\title{
The sonographic morphology of musculus palmaris longus in humans
}

\author{
K. Dąbrowski ${ }^{1}$, H. Stankiewicz-Jóźwicka ${ }^{3}$, A. Kowalczyk¹ ${ }^{1}$ M. Markuszewski ${ }^{4}$, B. Ciszek¹, 2 \\ ${ }^{1}$ Department of Descriptive and Clinical Anatomy, Medical University of Warsaw, Poland \\ 2Department of Neurosurgery in Bogdanowicz Children's Hospital, Warsaw, Poland \\ ${ }^{3}$ Department of Piano, Harpsichord and Organ, Fryderyk Chopin University of Music, Warsaw, Poland \\ ${ }^{4}$ Department of Choir Conducting, Music Education, Church Music, Rhythmics and Dance, \\ Fryderyk Chopin University of Music, Warsaw, Poland
}

[Received: 16 November 2017; Accepted: 23 November 2017]

\begin{abstract}
Background: The aim of this work was to describe morphology and morphometry of musculus palmaris longus and compare the outcome of Shaeffer's test with ultrasound imaging.

Materials and methods: Forty forearms of 20 healthy volunteers $(11$ females, 9 males) were tested by Shaeffer's test and ultrasound imaging. Anthropological measurements of the forearm and ultrasound guided measurements of musculus palmaris longus were taken. The outcome was tested for statistical significance by Fisher's test.

Results: The examination revealed agenesis of palmaris longus in 6 cases, as well as 6 muscles showing quality variations. The Shaeffer's test gave 4 false-negative results. 28 muscles were described as spindle-shaped and 8 as pennated or bipennated. However, all the spindle-shaped muscles demonstrated a tendon going inside of the muscle's belly ranging from $2 \mathrm{~cm}$ to $11.5 \mathrm{~cm}$. The relation between the circumference of the forearm right below the elbow (mean: $15.38 \mathrm{~cm}$, SD: $1.83 \mathrm{~cm}$ ) and the approximated volume of the palmaris longus muscle's belly (mean: $4.72 \mathrm{~cm}^{3}, S D: 1.57 \mathrm{~cm}^{3}$ ) proved to be statistically significant (Fisher's test p-value $<0.05$ ).

Conclusions: Uncommon morphological variations have been shown. Spindle-shaped muscles have proved to have their tendons continued inside them. Palmaris longus muscle's belly has proved to take significant amount of volume within the proximal forearm. Shaeffer's test has shown to have $10 \%$ false-negative ratio. (Folia Morphol 2018; 77, 3: 509-513)
\end{abstract}

Key words: variability, palmaris longus muscle, hand, wrist, forearm, Shaeffer's test

\section{INTRODUCTION}

Musculus palmaris longus ( $\mathrm{PL}$ ) is usually being described as a spindle-shaped, functionally redundant, wrist flexing muscle lying in the anterior compartment of the forearm. It's most typical insertions are medial epicondyle of humerus, and palmar aponeurosis [1].
PL presents very high morphological variability. The most common variation is its absence which seems to depend on ethnicity and range from $0.17 \%$ in Nigerians to $63.9 \%$ in Turkish and Indian population $[4,9,19,21]$. Its absence or presence doesn't seem to have any impact on biomechanical functions of the 


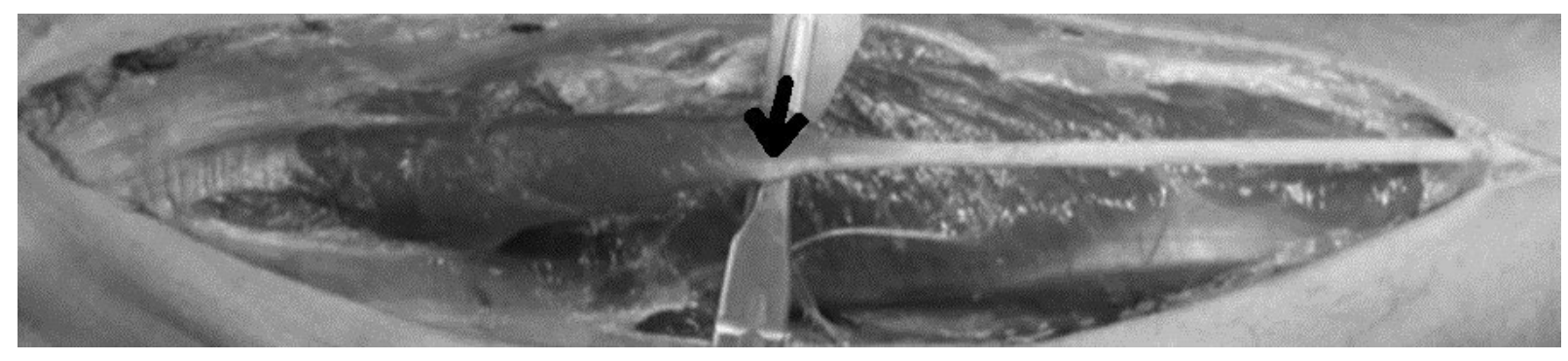

Figure 1. Seemingly spindle-shaped palmaris longus on the fresh cadaver. However, it is noticeable how a tendon goes within the muscle's belly (arrow). The courtesy of Forensic Medicine Department of the Medical University of Warsaw.

upper limb $[3,14]$. Due to that, and due to being fully developed at birth unlike other potential options, PL is commonly used in tendon grafts, lip and chin restorations, ptosis corrections, eyelid defect and facial paralysis treatment as well as other plastic surgery procedures. It is also used as an important landmark, and for genetic and anthropological studies [1, 9, $12,20]$. Attachment of the PL to the aponeurosis palmaris may improve tension of the aponeurosis which is necessary for adequate grasp of the hand and fingers (Fig. 1).

\section{MATERIALS AND METHODS}

The study was performed on 20 healthy volunteers between 19 and 26 years of age ( 11 females, 9 males). Their upper limb anthropological measurements were taken, including arm, forearm and hand length, general upper limb length and forearm circumference at thickest and thinnest points. Their PL morphology was tested first by Shaeffer's test and then by ultrasound (US) imaging. US guided measurements of the PL were taken. The measurements consisted of the length of the entire structure, muscle's belly, muscle's tendon and tendon within the belly, thickness and width of the tendon and maximal thickness and width of the muscle's belly. For the sake of calculating the muscle's belly volume, the shape of muscle's belly was approximated to an octahedron.

The US machine used during the examination was LOGIQ F8 GE with L6-12, 6-13 MHz probe.

\section{RESULTS}

Sixteen out of 20 examined subjects showed bilateral presence of $\mathrm{PL}, 2$ demonstrated unilateral absence (left hand, both male) and 2 - bilateral absence ( 1 male, 1 female), which sums up to 34 muscles. The mean length of the entire structure was $27.41 \mathrm{~cm}$ (female range: $25-28 \mathrm{~cm}$ left, $14-28 \mathrm{~cm}$ right; male range: $26-33 \mathrm{~cm}$ left, $27-34$ right). The mean circumference at the level of the wrist was $15.94 \mathrm{~cm}$ (female range: $14.5-17 \mathrm{~cm}$ left, $14.5-17 \mathrm{~cm}$ right; male range: $15.5-18 \mathrm{~cm}$ left, $16-18 \mathrm{~cm}$ right). The mean circumference at the thickest level was $25.89 \mathrm{~cm}$ (female range: $21.5-27.5 \mathrm{~cm}$ left, $22.5-27.5 \mathrm{~cm}$ right; male range: $24.5-30.5 \mathrm{~cm}$ left, $24-31 \mathrm{~cm}$ right).

The mean of approximate muscle's belly volume was $4.72 \mathrm{~cm}^{3}$ with length: $14.77 \mathrm{~cm}$, width: $1.95 \mathrm{~cm}$ and thickness: $0.64 \mathrm{~cm}$ (female: mean volume $=$ $=3.71 \mathrm{~cm}^{3}$ left, $3.84 \mathrm{~cm}^{3}$ right, mean length $=14.1 \mathrm{~cm}$ left, $14 \mathrm{~cm}$ right, mean width $=1.71 \mathrm{~cm}$ left, $1.81 \mathrm{~cm}$ right, mean thickness $=0.58 \mathrm{~cm}$ left and right. male: mean volume $=6.68 \mathrm{~cm}^{3}$ left, $5.98 \mathrm{~cm}^{3}$ right; mean length $=15.67 \mathrm{~cm}$ left, $15.94 \mathrm{~cm}$ right, mean width $=2.15 \mathrm{~cm}$ left, $2.30 \mathrm{~cm}$ right, mean thickness = $=0.79 \mathrm{~cm}$ left, $0.66 \mathrm{~cm}$ right).

Twenty-eight out of 34 examined muscles were recognised as spindle-shaped, with one being inverted (male, right, bilateral PL) and two presenting ramified tendon within the belly (female, bilateral variation). Six out of 34 muscles were recognised as pennated or bipennated muscles. One of the specimens (female, bilateral absence) showed a tendon connecting aponeurosis palmaris with musculus flexor digitorum superficialis (right). One of the specimens (female, bilateral presence) demonstrated a bipennated PL (right) with proximal insertion being aponeurosis antebrachii, around half way through the length of the forearm. Detailed analysis of sonography indicated that in all of the tested PL muscles the tendon was continued inside the muscle's belly.

The Shaeffer's test compared to ultrasound imaging gave 30 true-positive results, 6 true-negative results and 4 false-negative results. There were no false-positive Shaeffer's test results (Table 1). 
Table 1. Relation of the forearm circumference and approximated volume of the palmaris longus (PL) muscle's belly. To avoid confounding variables only volunteers with bilateral PL presence has been chosen

\begin{tabular}{|c|c|c|c|c|c|c|c|}
\hline \multirow[t]{2}{*}{ Mean } & & \multicolumn{2}{|c|}{ Left } & \multicolumn{2}{|c|}{ Right } & \multicolumn{2}{|c|}{ Both } \\
\hline & & SD & Mean & SD & Mean & SD & \\
\hline \multirow[t]{3}{*}{ Forearm circumference $[\mathrm{cm}]$} & Male & 27.67 & 2.52 & 27.92 & 2.84 & 27.79 & 2.67 \\
\hline & Female & 23.85 & 1.51 & 24.1 & 1.27 & 23.98 & 1.37 \\
\hline & Both & 25.28 & 2.67 & 25.53 & 2.7 & 25.41 & 2.67 \\
\hline \multirow[t]{3}{*}{ PL's belly length [cm] } & Male & 15.67 & 2.88 & 15.08 & 2.33 & 15.38 & 1.83 \\
\hline & Female & 14.1 & 3.07 & 14 & 2.64 & 14.05 & 2.81 \\
\hline & Both & 14.89 & 3.01 & 14.41 & 2.51 & 14.55 & 2.51 \\
\hline \multirow[t]{3}{*}{ PL's belly width $[\mathrm{cm}]$} & Male & 2.15 & 0.49 & 2.41 & 0.41 & 2.29 & 0.35 \\
\hline & Female & 1.71 & 0.31 & 1.81 & 0.23 & 1.76 & 0.23 \\
\hline & Both & 1.88 & 0.42 & 2.04 & 0.42 & 1.96 & 0.38 \\
\hline \multirow[t]{3}{*}{ PL's belly thickness [cm] } & Male & 0.79 & 0.16 & 0.64 & 0.21 & 0.71 & 0.17 \\
\hline & Female & 0.58 & 0.09 & 0.58 & 0.11 & 0.58 & 0.06 \\
\hline & Both & 0.66 & 0.15 & 0.6 & 0.15 & 0.66 & 0.13 \\
\hline \multirow[t]{3}{*}{ PL's belly volume $\left[\mathrm{cm}^{3}\right]$} & Male & 6.68 & 0.98 & 5.89 & 1.61 & 6.28 & 0.84 \\
\hline & Female & 3.71 & 1.41 & 3.85 & 1.09 & 3.78 & 1.04 \\
\hline & Both & 4.83 & 1.93 & 4.61 & 1.62 & 4.72 & 1.57 \\
\hline
\end{tabular}

Fisher's test $\mathrm{p}$-value $=0.04661 ; \mathrm{SD}-$ standard deviation

\section{DISCUSSION}

The US guided measurements of the tendons and bellies of the examined muscles allowed a rough estimation of PL muscle volume. Fisher's statistical significance test was performed on the mean volume (mean of the sides) of the muscle's belly and mean circumference of the forearm right below the elbow of the specimen with bilateral PL presence. The correlation proved to be significant $(p<0.05)$ which could mean that PL develops alongside all the other muscles of the forearm, which would be very unlikely if it did not participate in the manual activities alongside them. It also shows that PL, no matter how small the belly, takes significant volume in the proximal part of the forearm.

As mentioned above, PL is described as a spindle-shaped muscle, with tendon ending at the beginning of the muscle's belly. It is believed to be characteristic for humans and old world primates. A pennated or bipennated muscle is believed to be domain of lemuriformes and new world primates [19]. Meanwhile, during the examination it was observed that not only 6 of the examined human muscles showed a pennated or bipennated structure, but also all the spindle-shaped muscles presented a tendon within the muscle's belly, ranging between $2 \mathrm{~cm}$ and $11.5 \mathrm{~cm}$ of length. The Caucasian model of the PL was described by Loth [13] - with the length of the tendon dominating the length of the muscle's belly. However, the part of the

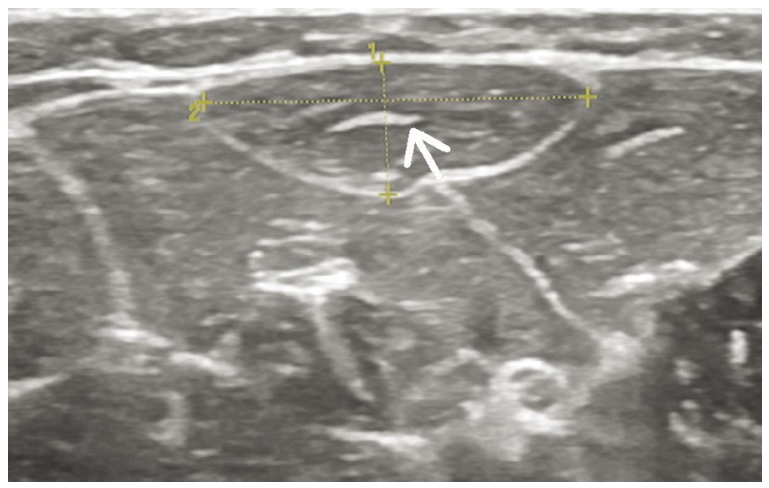

Figure 2. Ultrasound guided measurements (dotted lines) of the width (2) and thickness (1) of the palmaris longus (PL) muscle's belly. In the middle (arrow) a visible PL tendon within the belly.

tendon inside the muscle's belly may play important role in biomechanics of the muscle. If the tendon is identified inside of morphologically spindle-shaped muscle it is pennated or bipennated muscle. It also influences surgical destination of PL. In some cases researchers consider the end of muscle's belly the real beginning of the tendon [7]. But the tendon in muscle's belly may also be valuable as graft material, as well as of clinical importance, especially when it comes to musculotendinous junction pathology like it happens in other muscles with important part of tendon inside the belly (Fig. 2) [6].

Despite its potential unreliability, Shaeffer's test is still vastly used both in clinic and in the research, even 
by authors who criticise it in their publications, mostly due to its simplicity $[9,11,12,15,17,20]$. In some situations, to avoid the above mentioned unreliability, authors tend to complement Shaeffer's test with other clinical tests such as Pushpakumar's, Mishra's, Thompson's or Gangata [1, 9, 11, 12, 20]; however, in most cases the differences between tests are marginal changes in fingers positioning or pressure applying [17]. It seems reasonable to question such methods as potentially susceptible to human error. That's why in this study Shaeffer's test was confronted with US imaging. As it turned out, the Shaeffer's test gave a false-negative results four times - twice with spindle-shaped muscle and twice with bipennated muscle. In 1 case (female, age 20, right hand) a pennated muscle's belly was present on the entire length of the tendon, which might have blurred the outline of the structure. In another case (male, age 20, left hand, spindle-shaped) the PL tendon near the wrist seemed barely visible in the US imaging, which might suggest tendon's internal structure's peculiarity. Coincidentally, on the other hand of one of the four specimen mentioned before, Shaeffer's test proved to be truepositive despite the $\mathrm{PL}$ tendon running much further on the radial side of the wrist than in the typical case - variability that would hinder PL function as a landmark. In that case there would be need for relying solely on bone structure landmark palpation [2]. Inverted $\mathrm{PL}$, which needs to be brought to attention due to its correlation with median nerve compression [4], has been marked as a true-positive in Shaeffer's test. The observed variations, including barely visible tendon, ramified tendon and inverted $\mathrm{PL}$, fall into the category of quality variations and have been observed and described before $[8,18]$. Some of the observed variations, like agenesis or fascial insertions, have also been described in the lower limb analogue of $\mathrm{PL}$ - the plantar muscle [16]. During the examination, multiple cases of unusually prominent tendons of wrist and digit flexors were observed, ones that could be mistaken for the positive Shaeffer's test result. Overall, it seems that clinical tests for the presence of PL might be affected by other, less frequent variabilities of the muscle as well as a simple human error (Fig. 3) [17].

It is still unknown what determines whether PL will be present or absent. There is some research pointing out a correlation between its presence in parents and their children. Such observations of unilateral and bilateral presence of PL between generations brought the authors to conclusion that gene or set of

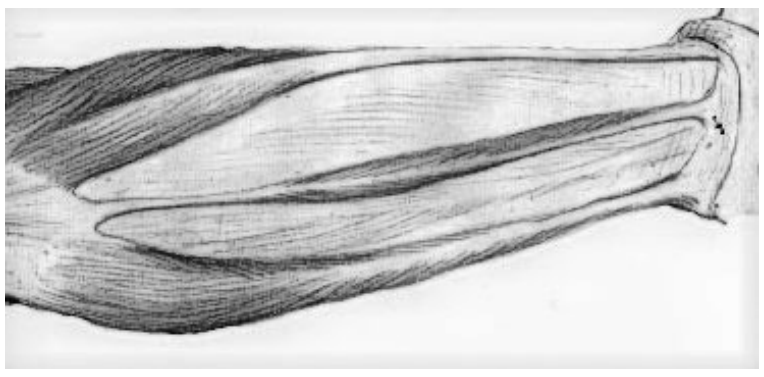

Figure 3. A depiction of the inverted palmaris longus. The graphic from the collection of The Department of Descriptive and Clinical Anatomy, Medical University of Warsaw.

genes responsible for $\mathrm{PL}$ presence would be dominant $[9,10,18]$, although Loth [13] describes PL absence as dominant feature, at least in white population. Other researchers, focused on relations between humans and different types of primates, argue that those relations would also suggest a recessive set of genes [19]. One of the theories used to explaining much less common agenesis in African population compared to EuroAsian groups draws correlation between prevalence of PL and type of labour performed most commonly by specific ethnic groups throughout the ages, specifically manual labour in Africa $[12,15]$. Undoubtedly, anthropological studies need to be backed by local history research as proven by experiment in Dercen - an isolated Ukrainian village of Hungarian origin, which revealed unusual pattern - among people born before 1945 over $70 \%$ showed PL agenesis with over $50 \%$ being bilateral absence. Among people born after 1945 less than $48 \%$ demonstrated PL agenesis from which only $28 \%$ being bilateral absence. This example reveals how a unique morphological pattern can be irreversibly disturbed by single historical event, the event being Soviet occupation in 1944-1945 [5]. This change is even more characteristic when noted that $\mathrm{XIX}$ century research describes PL as absent in only $10.7 \%$ of Russian male population [13].

Most authors seems to agree that PL agenesis frequency depends on ethnicity of the test subjects and that unilateral absence is about twice as often as bilateral absence $[11,12,20,21]$. This study, performed on young adults from various sites of Poland, does not fall into described model, nor it follows the frequency of agenesis characteristic for Polish population (being $15 \%$ instead of expected $8.6 \%$ ), falling closer to Jewish (19.5\%) or generalised EuropeanCaucasian (15.2\%) [11]. This, however, might be due to limited number of specimens examined. 


\section{CONCLUSIONS}

The study has shown uncommon morphological variations of musculus $\mathrm{PL}$, especially presence of pennated and bipennated type of muscle's belly in humans. Even in spindle-shaped PL the tendon is continued inside of belly. The belly of PL takes significant part of volume of the proximal forearm. Apart from that confronting clinical Shaeffer's test with US imaging has shown $10 \%$ ratio of false-negative Shaeffer's test which needs to be considered when deciding the transplant material in tendon grafts and reconstruction procedures.

\section{REFERENCES}

1. Agarwal P. Absence of the palmaris longus tendon in Indian population. Indian J Orthop. 2010; 44(2): 212-215, doi: 10.4103/0019-5413.61863, indexed in Pubmed: 20419011.

2. Ajayi NO, Naidoo N, Lazarus L, et al. Determination of the median nerve safe-zone in the carpal tunnel using the distal forearm bony prominences. Folia Morphol. 2014; 73(4): 409-413, doi: 10.5603/FM.2014.0062, indexed in Pubmed: 25448897.

3. Angelini Júnior LC, Angelini FB, de Oliveira BC, et al. Use of the tendon of the palmaris longus muscle in surgical procedures: study on cadavers. Acta Ortop Bras. 2012; 20(4): 226-229, doi: 10.1590/S1413-78522012000400007, indexed in Pubmed: 24453608.

4. Barkáts N. Hypertrophy of palmaris longus muscle, a rare anatomic aberration. Folia Morphol. 2015; 74(2): 262-264, doi: 10.5603/FM.2015.0040, indexed in Pubmed: 26050817.

5. Barkáts N. Change of the agenesis rate of palmaris longus muscle in an isolated village in Ukraine. Folia Morphol. 2015; 74(4): 470-474, doi: 10.5603/FM.2015.0109, indexed in Pubmed: 26620507.

6. Bogacka U, Dziedzic D, Komarnitki I, et al. Anatomy of the long peroneal muscle of the leg. Folia Morphol. 2017; 76(2): 284-288, doi: 10.5603/FM.a2016.0054, indexed in Pubmed: 27714727.

7. Cerda A, Del Sol M. Morphology and morphometry of the tendons of the palmaris longus and radial carpal extensor muscles in humans. Int J Morphol. 2016; 34(4): 1515-1521, doi: 10.4067/s0717-95022016000400054.
8. Ciszek B, Szaro P. Palmaris longus muscle - case report and review. Acta Clinica. 2003; 3(4): 363-370.

9. Deniz M, Yldiz E. The prevalence and familial tendency of absence of the Palmaris Longus muscle in Turkish population Pak J Med Sci. 2011; 27(4): 754-758.

10. Gangata $\mathrm{H}$. The clinical surface anatomy anomalies of the palmaris longus muscle in the Black African population of Zimbabwe and a proposed new testing technique. Clin Anat. 2009; 22(2): 230-235, doi: 10.1002/ca.20751.

11. Hussain FN, Hasan T. Prevalence of congenital absence of Palmaris Longus tendon in young Jizani population of Saudi Arabia: A cross sectional study. Pak J Med Sci. 2012; 28(5): 865-869.

12. Kigera J, Mukwaya S. Frequency of agenesis palmaris longus through clinical examination - An East African Study. PLoS ONE. 2011; 6(12): e28997.

13. Loth A. Anthropologie des parties molles, Mianowski's Foundation. Masson \& Cie Warszawa - Paris. 1931.

14. Marpalli S, Bhat A, Gadahad M. A Case Reverse Palmaris Longus Muscle - An Additional Muscle in the Anterior Compartment of the Forearm. J Clin Diagnostic Res. 2016; 10(3): AD03-AD04.

15. Oladipo Gabriel S, Didia Blessing C, Ugboma A. Frequency of agenesis of Palmaris Longus Muscle in Nigerians. Int J Biol Anthropol. 2008; 3(2).

16. Olewnik $t$. The report on the co-occurrence of two different rate anatomic variations of the plantaris muscle tendon on both sides of an individual. Folia Morphol. 2017; 76(2): 331-333.

17. Pękala PA. Congenital absence of the palmaris longus muscle: A meta-analysis comparing cadaveris and functional studies. JPRAS. 2017; 70(12): 1715-1724.

18. Pires L, Perissé JP, Araújo G, et al. Hypertrophic reversed palmaris longus muscle: a cadaveric finding. Folia Morphol. 2018; 77(2): 403-405, doi: 10.5603/fm.a2017.0102.

19. Roqueline AGMF, Aversi-Ferreira TA, et al. Morphometric and statistical analysis of the palmaris longus muscle in human and non-human primates. Biomed Res Int. 2014; 2014: 178906, doi: $10.1155 / 2014 / 178906$, indexed in Pubmed: 24860810.

20. Syaiful A, Dody P. Palmaris longus absence in six largest Indonesian ethnicities and its relationship with gender, ethnicity, and hand dominance. Med J Indonesia. 2015: 24, doi: 10.13181/mji.v24i1.1191.

21. Thompson NW, Mockford BJ, Cran GW. Absence of the palmaris longus muscle: a population study. Ulster Med J. 2001; 70(1): 22-24, indexed in Pubmed: 11428320. 